\title{
Functional Imaging of Human Epidermal Growth Factor Receptor 2-Positive Breast Cancers and a Note about NOTA
}

\author{
Joanne E. Mortimer and John E. Shively \\ City of Hope Comprehensive Cancer Center/Beckman Research Institute, Duarte, California
}

\section{See the associated article on page 26.}

\section{B} $20 \%-30 \%$ of these cancers overexpress the human epidermal growth factor receptor 2 (HER2) protein, making them candidates for HER2-directed therapies. Trastuzumab (Herceptin; Roche), the first HER2-directed therapy, is a humanized monoclonal antibody that binds to the extracellular domain of HER2, preventing downstream signaling and cell proliferation. Trastuzumab is also an immunologic agent, stimulating antibody-dependent cytotoxicity. Investigators have developed radiolabeled trastuzumab as a PET imaging agent for use in HER2-positive breast cancer patients. However, its clinical role has yet to be established. In this issue of The Journal of Nuclear Medicine, Dr. Woo and his colleagues report the use of NOTA as a chelator for ${ }^{64} \mathrm{Cu}$ labeling of trastuzumab and claim more favorable pharmacokinetics than ${ }^{64} \mathrm{Cu}-$ DOTA-trastuzumab (1). But is NOTA a better chelator for ${ }^{64} \mathrm{Cu}$ than DOTA? Woo et al. base their comparison on a report by Paudyal et al. (2) in which the uptake of ${ }^{64} \mathrm{Cu}$-DOTA-trastuzumab in the liver was $26.9 \pm 7.4 \% \mathrm{ID} / \mathrm{g}$ at $24 \mathrm{~h}(3)$, whereas in contrast, the uptake of ${ }^{64} \mathrm{Cu}-\mathrm{NOTA}$-trastuzumab was $5.44 \pm$ 1.84 percentage injected dose per gram in the liver at $24 \mathrm{~h}$ (Fig. 4 in Woo et al.; (1)). They conclude that the difference in liver uptake between the 2 studies was due to release of ${ }^{64} \mathrm{Cu}$ from the DOTA but not from the NOTA chelate. However, the 2 studies cannot be compared because they were not performed using the same tumor models. Paudyal et al. (2) performed PET imaging on HER2-positive (HER2+) non-small cell xenografts, whereas Woo et al. performed PET imaging on HER2+ breast cancer xenografts. The difference in liver uptake may simply reflect differential shedding of HER2 antigen to the liver between the 2 types of tumors. To make their point, Woo et al. should have compared DOTA- versus NOTA-conjugated trastuzumab in the same tumor model. This is a clear example of confusing the chemical stability of metal chelates with their metabolic clearance in different tumor models.

\footnotetext{
Received Nov. 13, 2018; revision accepted Nov. 14, 2018.

For correspondence or reprints contact: Joanne Mortimer, City of Hope Comprehensive Cancer Center/Beckman Research Institute, 1500 East Duarte Rd., Duarte, CA 91010.

E-mail:jmortimer@coh.org

COPYRIGHT (C) 2019 by the Society of Nuclear Medicine and Molecular Imaging. DOI: 10.2967/jnumed.118.220905
}

\section{ASSESSMENT OF HER2 STATUS}

Candidacy for HER2-directed therapy is determined pathologically by overexpression of the HER2 protein, assessed by immunohistochemical staining or HER2 gene amplification using fluorescence in situ hybridization (FISH). The addition of HER2directed therapy to conventional chemotherapy is associated with improved response rates and overall survival for women with all stages of HER2 + breast cancer (4). Therefore, an accurate determination of HER2 status is critical for treatment decision making. Currently, HER2 status for each patient is determined pathologically on a small specimen obtained from a larger tumor in an individual who may have multiple disease sites.

Only 5\% of women present with de novo metastatic breast cancer. Most with metastatic disease present after having been treated for curative intent with surgery and systemic therapy, with or without radiation. In this setting, HER2 status is assessed on a recurrent or metastatic site and may differ from the original primary tumor in up to $30 \%$ of cases (5). Whether this change is attributed to tumor heterogeneity or to alterations induced by prior therapy, repeated assessment of HER2 status is important in determining appropriate treatment and currently requires a tumor biopsy. The pathologic criteria for defining HER2 + has changed over time and is updated regularly by a joint committee of the American Society of Clinical Oncology and College of American Pathology (6). The current determination of HER2 in clinical care has many shortcomings.

Some investigators claim that trastuzumab-labeled PET imaging can be used to "determine HER2 status." We correlated a relationship between HER2 assessment by FISH and ${ }^{64} \mathrm{Cu}-$ DOTA-trastuzumab PET uptake in biopsy-proven metastatic disease, and Sasada correlated ${ }^{64} \mathrm{Cu}$-DOTA-trastuzumab PET uptake in the primary tumor with $\operatorname{HER} 2$ assessment by $\operatorname{FISH}(7,8)$. Although trastuzumab-labeled PET imaging may provide an indirect assessment of HER2 status, it provides far more important information than HER2 status. It tells us that trastuzumab is being delivered to the tumor and at which sites. It is this information that patients and their oncologists need to know and information that cannot be gleaned from the pathologic assessment of HER2 from a single biopsy.

\section{PREDICTING TREATMENT EFFICACY}

Because trastuzumab-labeled PET provides an in vivo assessment of tumor uptake by trastuzumab, can we use it at baseline to predict for subsequent response to HER2-directed therapies? For trastuzumab PET to move into the clinic, it is critical to appreciate that trastuzumab as a single agent has only modest antitumor 


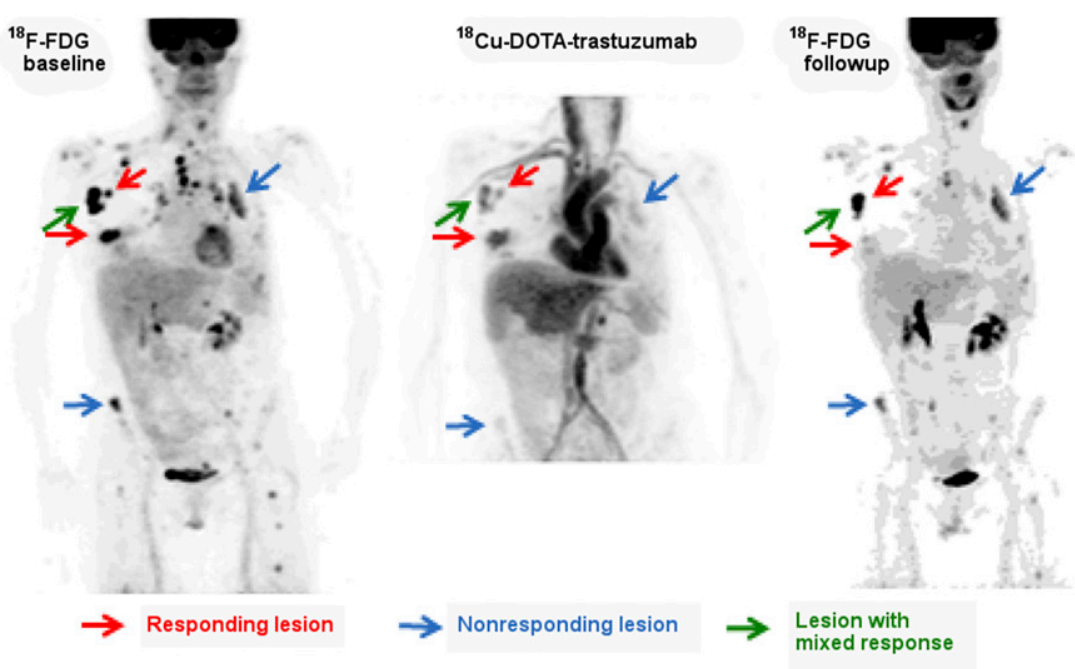

FIGURE 1. ${ }^{18} \mathrm{~F}$-FDG PET (left) and ${ }^{64} \mathrm{Cu}$-DOTA-trastuzumab PET (middle) were obtained before TDM-1. Posttreatment ${ }^{18} \mathrm{~F}-\mathrm{FDG}$ PET (right) shows tumor regression only in tumor sites imaged on ${ }^{64} \mathrm{Cu}$-DOTA-trastuzumab PET.

efficacy, with objective responses observed in only $15 \%$ of patients (9). However, when trastuzumab is coadministered with chemotherapy, the response rate and overall survival are significantly increased compared with chemotherapy alone (10). Because trastuzumab is virtually always given with chemotherapy, a pretreatment trastuzumab PET scan does not provide information about the individual benefit of trastuzumab.

Some investigators have used trastuzumab PET imaging in women who are already actively receiving treatment with trastuzumab, a drug with a 21-d half-life. Trastuzumab PET imaging in this setting is unlikely to provide useful clinical information as the tumor HER2 receptors may already be bound by trastuzumab, precluding uptake by the labeled trastuzumab. The most valuable use of trastuzumab PET is in newly diagnosed patients, women with newly relapsed disease, those who have been off the drug for more than $4-6 \mathrm{wk}$, and those treated with ado-trastuzumab emtansine (TDM-1, Kadcycla; Roche).

TDM-1 is an antibody-drug conjugate of trastuzumab and the cytotoxic agent maytansine. Its efficacy is directly related to tumor uptake of trastuzumab, which is exactly what labeled trastuzumab PET assesses. In the ZEPHIR trial from The Netherlands, baseline ${ }^{18} \mathrm{~F}$-FDG PET and ${ }^{89} \mathrm{Zr}$-labeled trastuzumab PET were performed in women with metastatic HER2 + breast cancer before TDM-1 treatment. ${ }^{18} \mathrm{~F}$-FDG PET was repeated before their second dose of TDM-1 to assess early response. Biopsy of metastatic disease was not an eligibility requirement, and more than one third of patients had received prior trastuzumab (although the interval from the last dose of trastuzumab is not reported). Of 55 evaluable patients, $29 \%$ did not show tumor uptake of ${ }^{89} \mathrm{Zr}$-labeled trastuzumab PET and many showed heterogeneous uptake at different metastatic sites. Importantly, ${ }^{89} \mathrm{Zr}$-labeled trastuzumab PET uptake predicted for improved time to TDM-1 treatment failure-15 versus 2.8 mo (11). The investigators continue to enroll patients and have included a biopsy in the expanded trial.

In our study, we used ${ }^{64} \mathrm{Cu}$-DOTA-traztuzumab to study women with metastatic HER2 + disease, and its potential clinical utility is exemplified by the following patient. A baseline ${ }^{18} \mathrm{~F}$-FDG PET scan identified multiple metastatic sites, and biopsy of a right axilla lesion confirmed HER2 positivity. Not all lesions identified on baseline ${ }^{18}$ F-FDG PET were identified on ${ }^{64} \mathrm{Cu}$-DOTAtrastuzumab PET (Fig. 1). After treatment with TDM-1, only the lesions visualized on ${ }^{64} \mathrm{Cu}$-trastuzumab PET responded to the antibody-drug conjugate. The combination of ${ }^{18}$ F-FDG PET with ${ }^{64} \mathrm{Cu}$-DOTA-trastuzumab PET demonstrates a heterogeneous uptake of trastuzumab, and with this knowledge, different chemotherapy added to TDM-1 might have provided tumor regression in the disease not imaged on ${ }^{64} \mathrm{Cu}$-DOTAtrastuzumab PET.

Currently ${ }^{18} \mathrm{~F}$-FDG PET is the only functional imaging agent used to stage breast cancer and may identify more metastatic lesions than conventional CT and bone scintigraphy. Radiolabeled trastuzumab imaging will not replace ${ }^{18}$ F-FDG PET, which is both sensitive and specific for all breast cancer subtypes. The greatest value of trastuzumab PET imaging is to provide an in vivo understanding of breast cancer biology that will ultimately help oncologists make better treatment decisions for patients.

\section{CENTRAL NERVOUS SYSTEM METASTASES}

Brain metastases are common in women with HER2+ breast cancer, and clinicians have questioned whether large molecules, such as humanized antibodies, are able to penetrate the bloodbrain barrier. Using ${ }^{64} \mathrm{Cu}$-DOTA-trastuzumab PET, we and others have demonstrated brain metastases in patients, suggesting that trastuzumab is taken up by brain metastases in some women (12). Ado-trastuzumab emtansine has been reported to have efficacy in women with HER2 + breast cancers who develop brain metastases (13). Not all patients in these series respond to TDM-1, and one would hypothesize that trastuzumab PET uptake could be used to identify patients for whom initial treatment with TDM-1 is appropriate. Systemic control of brain metastases with TDM-1 may delay further central nervous system progression and the need for brain irradiation with its associated toxicities. A clinical trial of trastuzumab PET to predict for benefit from TDM-1 in this setting is needed.

Is there an advantage of using ${ }^{64} \mathrm{Cu}$ - versus ${ }^{89} \mathrm{Zr}$-labeled trastuzumab PET imaging? Since both agents successfully demonstrate trastuzumab uptake in breast cancer, it is hard to pick one over the other, and a head-to-head comparison of these 2 agents is not a good use of patient resources at this time.

Before we are able to routinely incorporate trastuzumab PET imaging into the clinic, we need to know what the images are telling us. Is there an uptake value that predicts which patients are likely to benefit from TDM-1? Such studies will require close collaboration between all stakeholders-oncologists, nuclear medicine physicians, physicists, radiochemists, and patients. In addition, we cannot assume that trastuzumab PET will predict the antitumor efficacy of other HER2-directed agents such as pertuzumab, lapatinib, and neratinib, which have different mechanisms of action on the HER2 receptor. The ability to predict whether a single HER2-directed agent like TDM-1 will be effective in an individual patient at all sites of disease is the ultimate in precision medicine and has the potential to take trastuzumab PET imaging from a research tool into clinical care. 


\section{DISCLOSURE}

No potential conflict of interest relevant to this article was reported.

\section{REFERENCES}

1. Woo S-K, Jang SJ, Seo MJ, et al. Development of ${ }^{64} \mathrm{Cu}-\mathrm{NOTA}$-trastuzumab for HER2 targeting: radiopharmaceutical with improved pharmacokinetics for human study. J Nucl Med. 2018;60:26-33.

2. Paudyal P, Paudyal B, Hanaoka H, et al. Imaging and biodistribution of Her2/ neu expression in non-small cell lung cancer xenografts with ${ }^{64} \mathrm{Cu}$-labeled trastuzumab PET. Cancer Sci. 2010;101:1045-1050.

3. Paudyal B, Paudyal P, Oriuchi N, Hanaoka H, Tominaga H, Endo K. Positron emission tomography imaging and biodistribution of vascular endothelial growth factor with ${ }^{64} \mathrm{Cu}$-labeled bevacizumab in colorectal cancer xenografts. Cancer Sci. 2011;102:117-121.

4. Giordano SH, Temin S, Kirshner JJ, et al. Systemic therapy for patients with advanced human epidermal growth factor receptor 2-positive breast cancer: American Society of Clinical Oncology clinical practice guideline. J Clin Oncol. 2014;32:2078-2099.

5. Meng S, Tripathy D, Shete S, et al. HER-2 gene amplification can be acquired as breast cancer progresses. Proc Natl Acad Sci USA. 2004;101:9393-9398.

6. Wolff AC, Hammond MEH, Allison KH, et al. Human epidermal growth factor receptor 2 testing in breast cancer: American Society of Clinical Oncology/
College of American Pathologists clinical practice guideline focused update. J Clin Oncol. 2018;36:2105-2122.

7. Mortimer JE, Bading JR, Park JM, et al. Tumor uptake of ${ }^{64} \mathrm{Cu}$-DOTA-trastuzumab in patients with metastatic breast cancer. $J$ Nucl Med. 2018;59:38-43.

8. Sasada S, Kurihara H, Kinoshita T, et al. ${ }^{64} \mathrm{Cu}$-DOTA-trastuzumab PET imaging for HER2-specific primary lesions of breast cancer. Ann Oncol. 2017;28:20282029.

9. Cobleigh MA, Vogel CL, Tripathy D, et al. Multinational study of the efficacy and safety of humanized anti-HER2 monoclonal antibody in women who have HER2overexpressing metastatic breast cancer that has progressed after chemotherapy for metastatic disease. J Clin Oncol. 1999;17:2639-2648.

10. Slamon DJ, Clark GM, Wong SG, Levin WJ, Ullrich A, McGuire WL. Human breast cancer: correlation of relapse and survival with amplification of the HER2/neu oncogene. Science. 1987;235:177-182.

11. Gebhart G, Lamberts LE, Wimana Z, et al. Molecular imaging as a tool to investigate heterogeneity of advanced HER2-positive breast cancer and to predict patient outcome under trastuzumab emtansine (T-DM1): the ZEPHIR trial. Ann Oncol. 2016;27:619-624.

12. Kurihara $\mathrm{H}$, Hamada A, Yoshida M, et al. ${ }^{64} \mathrm{Cu}$-DOTA-trastuzumab PET imaging and HER2 specificity of brain metastases in HER2-positive breast cancer patients. EJNMMI Res. 2015;5:8.

13. de Vries CLG, Linn SC, Brandsma D. Response of symptomatic brain metastases from HER-2 overexpressing breast cancer with T-DM1. J Neurooncol. 2016;127:401-403. 